\title{
Procesos de Generalización y Pensamiento Algebraico ${ }^{1}$
}

\author{
Generalization Processes and Algebraic Thinking \\ Processos de generalização e pensamento algébrico \\ Recibido: mayo 2013 \\ Aceptado: agosto 2013 \\ Pedro Javier Rojas Garzón ${ }^{2}$ \\ Rodolfo Vergel Causado ${ }^{3}$
}

\section{Resumen}

En las dos sesiones programadas para este taller se abordará, por una parte, algunas actividades relacionadas con generalización de patrones figurales y/o numéricos, como recurso didáctico orientado a ubicar en los referentes teóricos elementos para el análisis de las producciones matemáticas de niños y jóvenes en torno a actividades como las antes mencionadas; por otra parte, actividades orientadas a posibilitar y potenciar conexiones entre conceptos de la matemática escolar asociados a los pensamientos numérico, métrico y variacional.

Palabras clave: Patrones, Generalización, pensamiento algebraico.

\begin{abstract}
In the two sessions scheduled for this workshop will address the one hand, some activities related to generalization of figural patterns and / or numeric, as a teaching resource designed to locate elements in the theoretical framework for the analysis of mathematical productions of children and youth around activities like those described above, on the other hand, activities to facilitate and enhance connections between school mathematics concepts associated with numerical thoughts, metric and variational.
\end{abstract}

Keywords: Patterns, Generalization, algebraic thinking.

\section{Resumo}

Nas duas sessões agendadas para este workshop irá abordar um lado, algumas atividades relacionadas à generalização de padrões figurativos eou numéricas, como recurso didático projetado para localizar os elementos no quadro teórico para a análise das produções matemáticas de crianças e jovens em torno de atividades como as descritas acima, por outro lado, as atividades para facilitar e melhorar as conexões entre os conceitos do ensino de matemática associados pensamentos numéricas, métricas e variacional.

Palavras-chave: padrões, a generalização, o pensamento algébrico.

1 Artículo de Investigación

2 Universidad Distrital Francisco José de Caldas, Bogotá-Colombia. Contacto: pedroedumat@udistrital.edu.co

3 Universidad Distrital Francisco José de Caldas, Bogotá-Colombia. Contacto: rodolfovergel@gmail.com 


\section{Introducción}

Es usual escuchar a estudiantes (y profesores) hablar sobre dificultades para aprender (y enseñar) álgebra, particularmente en grados $8^{\circ}$ ó $9^{\circ}$; dificultades asociadas al uso de "letras" y al significado que tienen dichas "letras" en contextos matemáticos. De hecho muchos estudiantes, que en los siete u ocho años anteriores habían tenido un buen desempeño en matemáticas, manifiestan su extrañeza de tener que trabajar con letras en matemáticas y se escuchan expresiones como: “... y por qué tengo que trabajar con letras”, o, “¿sí ve?, ¿de qué sirvió todo lo anterior? ... ipara que ahora no entienda nada!, o, incluso, "aparecieron las malditas letras". En trabajos como el de Kieran (1989), se reconoce precisamente que una dificultad asociada con el proceso de aprendizaje del álgebra escolar tiene que ver, precisamente, con el cambio en las formas de trabajar, pues los estudiantes deben enfrentarse a un cambio de las convenciones respecto al trabajo previo que venían realizando en aritmética. Sobre este aspecto, esta autora plantea que existen tres cambios significativos:

- la concatenación de símbolos,

- uso de paréntesis y

- usos del signo igual;

Además de reconocer otras dificultades, como la interpretación de las "letras" y el reconocimiento y uso de estructuras (superficial y sistémica).

\section{Ideas iniciales sobre la iniciación al álgebra escolar}

En el contexto colombiano el Grupo PRETEXTO, de la Facultad de Ciencias y Educación de la Universidad Distrital, realizó a mediados de los años noventa una investigación relacionada con la Transición aritmética-álgebra en la que, por un lado, se evidencian varias de las dificultades reportadas en el contexto internacional (ver, por ejemplo, Kieran, 1989) y, por otro, se reportan causas de incomprensión del concepto de variable, planteando algunos requerimientos para la comprensión de dicho concepto (Pretexto, 1996, 1999).
Veamos, a modo de contextualización, algunos ejemplos:

1. Concatenación de símbolos. En el contexto aritmético, concatenar dos símbolos, esto es, poner uno junto a otro, significa una suma: $25=20+5$ ó $25=2$ decenas +5 unidades; $3 \frac{1 / 4}{4}=$ $3+1 / 4$. En el contexto algebraico, concatenar dos símbolos significa multiplicar: $2 \mathrm{a}=2 \mathrm{xa}$. ¿Es descabellado pensar en que $2 \mathrm{a}=2+\mathrm{a}$ ó $2 \mathrm{a}$ $=20+\mathrm{a}$ ?

2. Uso de paréntesis. ¿A qué es igual $2+3 \times 4$ ? Usualmente los estudiantes plantean, al menos, dos respuestas diferentes: 20 y 14, obtenidas mediante los siguientes procedimientos: $(2+3) \mathrm{x} 4=5 \mathrm{x} 4=20 \quad \mathrm{y}$ $2+(3 \times 4)=2+12=14$. ¿Cuál de ellos es equivocado?, ¿por qué?

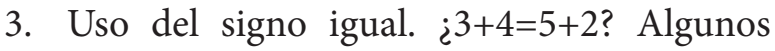
estudiantes responden que esa igualdad no es válida, porque $3+4$ no da 5 sino 7 ¿Qué explica esta respuesta?

Entre los aspectos reportados por el Grupo Pretexto, está el relacionado con la interpretación de las letras en contextos matemáticos, y la comprensión de la noción de variación y el concepto de variable. Entre las características asociadas con la variable están: (1) La variable pertenece siempre a un universo, y desde él debe ser interpretada, (2) El significado de variar que se le adjudica a la variable, corresponde al hecho que ella es representación, indistinta y simultánea de los distintos individuos que conforman su universo, (3) aparece siempre haciendo parte de una expresión, que da cuenta de la relación de dependencia que se desea destacar entre los individuos de su universo y (4) El universo al que pertenece la variable, sin ser tiempo, está implícitamente connotado de éste, en otras palabras, el tiempo se imbrica al universo de la variable, ajustándose a su cardinalidad y su estructura.

En relación con los aspectos anteriores, Pretexto señala la necesidad de reconocer qué universos numéricos tienen como referencia los estudiantes o en cuales tienen algún dominio. Plantea que un 
requerimiento para la comprensión del concepto de variable es contar con universos densos, sin embargo, a partir del trabajo que usualmente se les propone a los estudiantes -en los 7 u 8 primeros años de escolaridad formal-, esto no se logra. A pesar que los estudiantes tienen algunas experiencias con números enteros, con racionales y con algunos irracionales, básicamente sólo logran un dominio sobre los números naturales.

A modo de ilustración sobre los universos numéricos a los cuales "acuden" los estudiantes, destacamos el siguiente ejercicio propuesto por Pretexto (1997, p. 56):

a. Escriba en el cuadro las medidas de la base y la altura de cinco rectángulos distintos, cuya área sea $6 \mathrm{~cm} 2$

\begin{tabular}{|l|l|l|}
\hline \multicolumn{1}{|c|}{ Rectángulo } & Metida de la base & Medida de la altura \\
\hline $1^{\circ}$ & & \\
\hline $2^{\circ}$ & & \\
\hline $3^{\circ}$ & & \\
\hline $4^{\circ}$ & & \\
\hline $5^{\circ}$ & & \\
\hline
\end{tabular}

Fuente: Elaboración propia

b. Escriba las medidas de la base y la altura de tres rectángulos distintos, cuya área sea $3 \sqrt{2} \mathrm{~cm} 2$

\begin{tabular}{|l|l|l|}
\hline \multicolumn{1}{|c|}{ Rectingulo } & Melida de la base & Medida de la altura \\
\hline $1^{\circ}$ & & \\
\hline $2^{\circ}$ & & \\
\hline $3^{\circ}$ & & \\
\hline
\end{tabular}

Fuente: Elaboración propia

Para la primera parte del ejercicio anterior, los estudiantes rápidamente encuentran medidas, en centímetros, para los dos (ó cuatro) primeros rectángulos: 1 y 6 (ó 6 y 1), 2 y 3 (ó 3y 2), pero dudan y difícilmente encuentran medidas para los demás, en tanto se mantienen en el universo de los números naturales; incluso, algunos respondieron 5 y 1 , centrando su atención en la obtención del 6, dejando a un lado el contexto de la situación.
Por otra parte, los integrantes del Grupo Pretexto, plantean algunas consideraciones prácticas para el trabajo en la transición aritmética-álgebra, enfatizando en los procesos de generalización y de simbolización.

\section{Pensamiento algebraico y procesos de enseñanza}

En los currículos de muchos países es explícito el propósito de desarrollar, desde las matemáticas escolares, la capacidad de los niños y jóvenes para razonar algebraicamente, lo cual es usual abordar en los últimos cursos de educación básica (secundaria, 14-15 años), aunque no necesariamente con éxito. En las últimas dos décadas se ha desarrollado un número significativo de trabajos de investigación que dan cuenta de la posibilidad de abordar este propósito desde edades tempranas; lo cual ha hecho surgir nuevamente discusiones sobre la pertinencia de curricularizar los desarrollos teóricos al respecto. Un número significativo de investigadores plantea que dicha curricularización no sólo es posible sino necesaria (ver, por ejemplo, Kieran, 2006), y que a partir del contacto con experiencias significativas, desde la formación inicial en aritmética, es posible avanzar en la construcción de esquemas asociados al pensamiento algebraico. En esta dirección, parece necesaria una reflexión explícita orientada a superar concepciones sobre el álgebra escolar, su enseñanza y su aprendizaje, ampliamente asociadas de manera casi exclusiva con el dominio de un conjunto de procedimientos y técnicas para factorizar expresiones, simplificarlas y resolver ecuaciones. Al respecto, Kaput (2000) plantea que:

Se debe buscar que los docentes aprendan a construir oportunidades para el aprendizaje del razonamiento algebraico a partir de las restricciones que impone su sistema educativo y las fuentes documentales de que dispone (textos, Internet, currículo, etc.). En particular se debe ayudar al docente a que se centre en las formas como los estudiantes pueden acceder a la generalización de su propio pensamiento matemático, así como a expresar y justificar sus propias generalizaciones. 
Queremos resaltar que si bien, para muchos estudiantes y profesores, el trabajo algebraico está asociado a la incorporación de nuevos signos -letras-, esto se debe en parte a su desconocimiento sobre elementos teóricos y metodológicos, así como también a la ausencia de experiencias -ya sean de aprendizaje o de enseñanza-, que les permita reconocer, especialmente a los profesores, que el desarrollo del pensamiento algebraico puede tener lugar mucho antes de la aparición de dicho tipo de signos. Azarquiel (1993), por ejemplo, reconoce la necesidad de desarrollar procesos de pensamiento que permita a los estudiantes la construcción de un pensamiento algebraico, insistiendo en que la construcción de tal pensamiento tiene lugar a lo largo de un proceso paralelo y continuo dentro del trabajo aritmético y geométrico que se inicia en los primeros años. En el mismo sentido, Butto y Rojano (2000) plantean que los tiempos didácticos para el aprendizaje del álgebra son prolongados, por lo que es conveniente iniciar en edades tempranas, aprovechando las fuentes de significados que están presentes en los contenidos matemáticos de la educación primaria. Por tanto, puede afirmarse que existe un consenso en la comunidad de investigadores en didáctica del álgebra respecto a la necesidad de potenciar el desarrollo del pensamiento algebraico desde los primeros años de educación básica (primaria).

Para Godino \& Font (2000, p.8) el razonamiento algebraico,

Implica representar, generalizar y formalizar patrones y regularidades en cualquier aspecto de las matemáticas. A medida que se desarrolla este razonamiento, se va progresando en el uso del lenguaje y el simbolismo necesario para apoyar y comunicar [...], especialmente las ecuaciones, las variables y las funciones $[\ldots]$

En términos de este autor, algunas características del razonamiento algebraico que son sencillas de adquirir por los niños, y por tanto deben conocer los maestros en formación, son:

1. Los patrones o regularidades existen y aparecen de manera natural en las matemáticas.
Pueden ser reconocidos, ampliados, o generalizados.

2. El mismo patrón se puede encontrar en muchas formas diferentes. Los patrones se encuentran en situaciones físicas, geométricas y numéricas.

3. Podemos ser más eficaces al expresar las generalizaciones de patrones y relaciones usando símbolos.

4. Las variables son símbolos que se ponen en lugar de los números o de un cierto rango de números.

Las funciones son relaciones o reglas que asocian los elementos de un conjunto con los de otro, de manera que a cada elemento del primer conjunto le corresponde uno y sólo uno del segundo conjunto. Se pueden expresar en contextos reales mediante gráficas, fórmulas, tablas o enunciados (Godino, 2000, p. 10).

El pensamiento algebraico, como forma particular de reflexionar matemáticamente, es caracterizado por Radford (2006) mediante tres elementos interrelacionados:

- El sentido de indeterminancia (objetos básicos como: incógnitas, variables y parámetro; opuesto a la determinancia numérica)

- La analiticidad (como forma de trabajar los objetos indeterminados; reconocimiento del carácter operatorio de los objetos básicos).

- La designación simbólica de sus objetos (manera específica de nombrar o referir los objetos)

Este autor reconoce que los objetos matemáticos son objetos «generales», y la actividad matemática es esencialmente simbólica. Plantea, además, la necesidad de reflexionar explícitamente sobre la relación entre el desarrollo del pensamiento algebraico y los procesos de generalización. 
Desde lo planteado anteriormente se genera, como exigencia para el profesor, la necesidad de tomar conciencia sobre requerimientos para posibilitar el desarrollo del pensamiento algebraico, a través del diseño e implementación de actividades, en diferentes contextos, para potenciar en los estudiantes formas diferenciadas de pensamiento algebraico.

\section{Procesos de generalización}

Es importante destacar que la generalización, además de ser un proceso, puede ser caracterizada por los medios que los sujetos utilizan para "reconocer" dicha generalidad. En su actividad los sujetos no sólo requieren reconocer la generalidad sino también contar con formas de expresarla, como posibilidad de "actuar" u operar con ella. Esto plantea la necesidad de incorporar en el trabajo de aula actividades que potencien procesos de generalización; por ejemplo, a partir del reconocimiento de patrones en secuencias de figuras y en secuencias numéricas. Veamos una secuencia figural:

\section{$\begin{array}{llll}\text { Figura } 1 & \text { Figura } 2 & \text { Figura } 3 & \text { Figura } 4\end{array}$}

Radford (2006) reconoce tres tipos de generalización, o estratos de generalidad, caracterizados por los medios de expresión usados por los sujetos en su actividad, incluyendo movimientos, gestos, lenguaje natural:

1. Generalización Factual. Los medios de expresión usados son los gestos, los movimientos, la actividad perceptual y las palabras. Por ejemplo, un estudiante señala con su mirada, con su índice, realiza un movimiento con el lápiz, dice "aquí", vuelve a señalar y dice "más 2".

2. Generalización Contextual. Los gestos y las palabras son sustituidos por otros medios de expresión como frases "clave". Por ejemplo, el estudiante dice "arriba quito uno" ó "dos por la figura menos uno”.

3. Generalización Simbólica. Las frases "clave" son representadas por símbolos. Por ejemplo, mediante expresiones como: $\mathrm{n}+(\mathrm{n}-1)$ ó $2 \mathrm{n}-1$.
Entrando de manera directa particular, el trabajo con tareas sobre generalización de patrones figurales parece ser una de las estrategias para introducir el álgebra en la escuela, pues entre otros aspectos, posibilita a los estudiantes acercarse a situaciones de variación importantes para el desarrollo del pensamiento algebraico. Esto sugiere poner atención en los procesos que dan lugar a la emergencia del pensamiento algebraico en la escuela.

Los estudios llevados a cabo por Azarquiel (1993), desde un punto de vista cognitivo, establecen que el proceso de generalización requiere tres pasos bien diferenciados, a saber:

- la visión de la regularidad, la diferencia, la relación

- su exposición verbal, y

- su expresión escrita, de la manera más concisa posible.

En la perspectiva del "álgebra temprana", el reconocimiento de lo general desempeña un papel esencial como condición previa de la expresión. Queremos insistir en que estas formas de expresión son progresivas y evolucionan, desde movimientos, gestos, palabras, frases hasta la incorporación explícita de símbolos "inventados" y/o convencionales para "capturar" la generalidad y hacerla operativa.

\section{Una mirada desde documentos curriculares}

Queremos destacar algunos de los elementos planteados en los estándares curriculares propuestos por el Consejo Nacional de Profesores de Matemáticas de Norteamérica (NCTM) y los planteados por el Ministerio de Educación Nacional (MEN) de Colombia; de manera particular, resaltar qué posición asumen respecto a la pregunta sobre la posibilidad o la necesidad de trabajar álgebra en niveles tempranos, así como opciones explícitas para hacerlo.

Si bien, desde el NCTM (2000) no se plantea explícitamente el trabajo en los primeros grados de primaria de lo que algunos llaman "álgebra temprana”, sí reconocen la importancia de trabajar desde estos cursos actividades orientadas a la búsqueda de patrones, así como realizar experiencias significativas con números y sus propiedades, 
como fundamento para un trabajo posterior, comprensivo, con símbolos y expresiones algebraicas. Plantean que requiere prestar menos atención a la manipular símbolos, memorizar procedimientos y hacer práctica de repetición sobre resolución de ecuaciones, así como también el no enfatizar la resolución de problemas rutinarios de un solo paso, o problemas prototipo cuyo razonamiento no dependa claramente del estudiante. Así, plantean la necesidad de prestar más atención a identificar y usar relaciones funcionales, desarrollar y usar tablas, gráficas y reglas para describir situaciones, realizar interpretaciones entre diferentes representaciones (verbales, gráficas, numéricas, tabulares, figurales, simbólicas), además de proponer problemas abiertos y tareas ampliadas, en diferentes contextos, que incorporen el uso de métodos informales en la resolución de problemas e investigar $\mathrm{y}$ formular preguntas a partir de situaciones problema. Desde el NCTM se plantean expectativas específicas, por grupos de grados, desde preescolar hasta el Grado 12. Por ejemplo:

- PreKinder-Grado 2: Usar representaciones concretas, pictóricas y verbales para desarrollar una comprensión de notaciones "simbólicas", inventadas y convencionales (p. 90).

- Grados 3-5: Describir, extender y hacer generalizaciones acerca de patrones geométricos y numéricos; Representar y analizar patrones y funciones usando palabras, tablas y gráficas

- Grados 6-8: Representar, analizar y generalizar una variedad de patrones con tablas, gráficas, palabras y, cuando sea posible, reglas simbólicas.

- Grados 9-12: Generalizar patrones usando explícitamente funciones definidas y definidas recursivamente.

Desde los documentos curriculares de matemáticas (Lineamientos, 1998; Estándares Básicos, 2003), el Ministerio de Educación Nacional de Colombia propone nuevos elementos teóricos y metodológicos con el propósito de actualizar la estructura curricular de la educación matemática en nuestro país. En particular, en los Lineamientos Curriculares se identifican dos elementos importantes:

- la introducción de los diferentes tipos de Pensamiento Matemático: Numérico, Espacial, Métrico, Variacional y Aleatorio, y

- un llamado de atención sobre la importancia de implementar al interior del aula procesos como la modelación, comunicación, la resolución de problemas, el razonamiento y la ejercitación de procedimientos que permitan el aprendizaje de las matemáticas en contextos significativos para los alumnos.

En las recomendaciones del documento de Lineamientos se propone una restructuración conceptual y metodológica del álgebra escolar, enfatizada desde el pensamiento variacional, que ponga el acento en los procesos de generalización, la comunicación, la argumentación y la modelación de situaciones de cambio, como ejes fundamentales en la construcción del pensamiento algebraico. Motivan el estudio de la variación y el cambio, de las regularidades y la detección de los criterios que rigen esas regularidades o las reglas de formación para identificar el patrón que se repite periódicamente, como elementos asociados al pensamiento algebraico. Plantean sugerencias explícitas sobre actividades para desarrollar el pensamiento variacional desde los primeros niveles de la Educación Básica Primaria:

Analizar de qué manera cambia, aumenta o disminuye la forma o el valor en una secuencia o sucesión de figuras, números o letras; hacer conjeturas sobre la forma o el valor del siguiente término de la secuencia; procurar expresar ese término, o mejor los dos o tres términos siguientes, oralmente o por escrito, o por medio de dibujos y otras representaciones, e intentar formular un procedimiento, algoritmo o fórmula que permita reproducir el mismo patrón, calcular los siguientes términos, confirmar o refutar las conjeturas iniciales e intentar generalizarlas. 
Así mismo, en el documento de Estándares se reafirma esta noción de pensamiento variacional como eje fundamental para dar estructura y sentido al aprendizaje del pensamiento algebraico en la escuela, y se muestra cómo el desarrollo del pensamiento variacional se puede potenciar desde los primeros años de la educación básica, centrado en lo que podríamos llamar el estudio de las regularidades y patrones. Podríamos afirmar, por ahora, que el pensamiento variacional se entiende como una forma específica de pensar matemáticamente, orientada a la construcción de estructuras conceptuales que fundamentan el estudio de la variación y el cambio. Por su parte, el pensamiento algebraico refiere al conjunto de procesos, procedimientos y esquemas que dan forma y sentido al pensamiento variacional.

\section{Referencias}

Azarquiel, Grupo. (1993). Ideas y actividades para enseñar álgebra. Madrid: Síntesis.

Godino, J. \& Font, V. (2000). Razonamiento Algebraico y su Didáctica para maestros. Universidad de Granada. Recuperado de: http://ddm.ugr.es/personal/jdgodino/ manual/ralgebraico.pdf.

Rojas, P. et al. (1999). La transición aritmética-álgebra. Bogotá: Universidad Distrital Francsico José de Caldas-Gaia. Recuperado de: http://www.udistrital. edu.co:8080/documents/47902/262723/ LibroTransicion + Aritmetica-Algebra_ Grupo+MESCUD_U_Distrital_1999.pdf
Kaput, J. (2000). Transforming algebra from an engine of inequity to an engine of mathematical power by "algebrafying" the K-12 curriculum. Dartmouth, MA: National Center for Improving Student Learning and Achievement in Math \& Sci.

Kieran, C. (2006). Research on the learning and the teaching of algebra: A broadening of sources of meaning. En A. Gutiérrez \& P. Boero (Ed.) Handbook of research on the psychology of mathematics education: Past, present, future (pp. 11-49). Rotterdam, The Netherlands: Sense Publishers. Recuperado de: www. sensepublishers.com/media/457-handbook-of-research-on-the-psychology-of-mathematics-educationa.pdf

Ministerio de Educación Nacional (1998). Lineaminetos curriculares-Área Matemáticas. Bogotá: MEN. Recuperado de: http://www.mineducacion.gov.co/cvn/1665/ article-89869.html

Estándares básicos de competencias en Lenguaje, Matemáticas, Ciencias y Ciudadanas. Bogotá: MEN Recuperado de: http://www.mineducacion.gov.co/cvn/1665/ article-116042.html

Radford, L. (2006). Algebraic thinking and the generalization of patterns: A semiotic perspective, PME-NA, Vol. 1, 2-21. 